\title{
Power-Velocity and Power-Efficiency Implications in the Limitation of Ramp Incremental Cycle Ergometry: Reply to Morales-Alamo et al.
}

\author{
Carrie Ferguson, ${ }^{1}$ Daniel T. Cannon, ${ }^{2}$ Lindsey A. Wylde, ${ }^{1}$ Alan P. Benson, ${ }^{1}$ and (1) Harry B. Rossiter ${ }^{1,3}$ \\ ${ }^{1}$ School of Biomedical Sciences \& Multidisciplinary Cardiovascular Research Centre, Faculty of Biological Sciences, \\ University of Leeds, Leeds, United Kingdom; ${ }^{2}$ School of Exercise \& Nutritional Sciences, San Diego State University, San \\ Diego, California; and ${ }^{3}$ Rehabilitation Clinical Trials Center, Division of Respiratory \& Critical Care Physiology \& \\ Medicine, Los Angeles Biomedical Research Institute at Harbor-UCLA Medical Center, Torrance, California
}

TO THE EDITOR: We thank Morales-Alamo et al. (5) for raising important questions worthy of further discussion. A key concern of both our studies $(3,4)$ is whether the limit of tolerance (LoT) in ramp-incremental exercise is reached with a reserve in power producing capacity. To this end, we made three important measurements in endurance-trained men $\left(\dot{\mathrm{V}}_{\mathrm{O}_{2 \max }} 130 \pm\right.$ $32 \%$ predicted) immediately at LoT: power of hyperbolic cycling $(\sim 55 \mathrm{rpm}, 310 \pm 58 \mathrm{~W})$; power required by the task $(352 \pm 58 \mathrm{~W})$; and maximal isokinetic power, termed LoT $\mathrm{P}_{\text {ISO }}\left(80 \mathrm{rpm}, 391 \pm 72 \mathrm{~W}\right.$ ). LoT $\mathrm{P}_{\text {ISO }}$ was not different from the power required by the task, hence no reserve in maximal evocable power was evident. The fact that hyperbolic power at LoT was lower than task power is not the relevant comparison, and is likely related to dissipation of flywheel inertia. Importantly, peak power production at the crank was not different at LoT between $\sim 55$ and $80 \mathrm{rpm}$ (Fig. 2, $B-C$, in Ref. 3). Increasing cadence during the isokinetic switch to a velocity similar to the ramp incremental, achieved a small increase in mean power, but this was insufficient to exceed the task requirement $(P=0.116$, effect size $=0.81, \beta=0.73)$. Interestingly, this finding is similar to Morales-Alamo et al. (4), where mean isokinetic power $10 \mathrm{~s}$ after intolerance (318 \pm $55 \mathrm{~W}$ ) was only $10 \%$ greater than maximal incremental flywheel power $(290 \pm 36 \mathrm{~W})$.

We commend Morales-Alamo et al. (4) in their rapid occlusion and biopsy to preserve and measure muscle metabolic conditions at 10 and $60 \mathrm{~s}$ after LoT and show an intramuscular metabolic reserve. We were unable to reference this insightful work, because our manuscript was submitted before their study was published. Similarly, we found an intramuscular metabolic reserve at LoT, albeit during bilateral knee extension, where $<5 \%$ of quadriceps volume reached extreme metabolic conditions (2). Thus it is perhaps unsurprising that a small biopsy sample also found a reserve. Although we agree that intramuscular fatigue alone cannot explain task failure, a metabolic reserve is not evidence that the muscle is capable of being fully activated or increasing power generation at intolerance. To clarify, our volunteers were well-motivated and strongly encouraged throughout both ramp-incremental and isokinetic

Address for reprint requests and other correspondence: C. Ferguson, School of Biomedical Sciences \& Multidisciplinary Cardiovascular Research Centre, Faculty of Biological Sciences, Univ. of Leeds, Leeds LS2 9JT, UK (e-mail: c.ferguson@leeds.ac.uk). phases of the test and showed high test-retest reproducibility (3). Despite this, we found a $\sim 40 \%$ reduction in maximal evocable muscle activity and $\sim 10 \%$ lower activity-normalized isokinetic power, suggesting muscle metabolism and activation conspire to limit power to equal the task at LoT.

Morales-Alamo et al. raise an interesting question with their comment: "higher shortening speed requires more ATP." In isolated mouse fast-twitch fiber bundles, efficiency increases with velocity, peaking at about one-third peak velocity (1). Unfortunately, the entire velocity-efficiency curve is not characterized for cycling, but it seems reasonable that the small increase in power we observed between $\sim 55$ and $80 \mathrm{rpm}$ (3) may not necessitate a greater ATP turnover should efficiency increase.

Although we do not agree that at intolerance during whole body incremental exercise power can be greatly increased above the task requirement-both our studies $(3,4)$ provide evidence to the contrary-clearly there remains considerable work to uncover the integrated mechanisms limiting whole body exercise under incremental, constant, or self-paced conditions.

\section{DISCLOSURES}

No conflicts of interest, financial or otherwise, are declared by the author(s).

\section{AUTHOR CONTRIBUTIONS}

Author contributions: C.F. and H.B.R. drafted manuscript; C.F., D.T.C., L.A.W., A.P.B., and H.B.R. edited and revised manuscript; C.F., D.T.C., L.A.W., A.P.B., and H.B.R. approved final version of manuscript.

\section{REFERENCES}

1. Barclay CJ. Mechanical efficiency and fatigue of fast and slow muscles of the mouse. J Physiol 497: 781-794, 1996.

2. Cannon DT, Howe FA, Whipp BJ, Ward SA, McIntyre DJ, Ladroue C, Griffiths JR, Kemp GJ, Rossiter HB. Muscle metabolism and activation heterogeneity by combined $31 \mathrm{P}$ chemical shift and T2 imaging, and pulmonary $\mathrm{O}_{2}$ uptake during incremental knee-extensor exercise. $J$ Appl Physiol 115: 839-849, 2013.

3. Ferguson C, Wylde LA, Benson AP, Cannon DT, Rossiter HB. No reserve in isokinetic cycling power at intolerance during ramp incremental exercise in endurance-trained men. J Appl Physiol 120: 70-77, 2016.

4. Morales-Alamo D, Losa-Reyna J, Torres-Peralta R, Martin-Rincon M, Perez-Valera M, Curtelin D, Ponce-Gonzalez JG, Santana A, Calbet JA. What limits performance during whole-body incremental exercise to exhaustion in humans? J Physiol 593: 4631-4648, 2015.

5. Morales-Alamo D, Martin-Rincon M, Perez-Valera M, Marcora S, Calbet JAL. No functional reserve at exhaustion in endurance-trained men? J Appl Physiol; doi:10.1152/japplphysiol.01006.2015. 